\title{
CHAIN OF PRIME IDEALS IN FORMAL POWER SERIES RINGS
}

\author{
ADA MARIA DE S. DOERING AND YVES LEQUAIN
}

\begin{abstract}
Let $R$ be a Noetherian domain and $P$ a prime ideal of $R$. Then $R_{P}\left[\left[X_{1} \ldots, X_{n}\right]\right]$ has a maximal chain of prime ideals of length $r$ if and only if $R\left[\left[X_{1}, \ldots, X_{n}\right]\right]_{\left(P, X_{1} \ldots, X_{n}\right)}$ does, if and only if $R\left[X_{1} \ldots \ldots X_{n}\right]_{\left(P . X_{1} \ldots . X_{n}\right)}$ does.
\end{abstract}

1. Introduction. Let $R$ be a ring, $R\left[X_{1}, \ldots, X_{n}\right]$ the ring of polynomials in $n$ variables over $R$ and $R\left[\left[X_{1}, \ldots, X_{n}\right]\right]$ the ring of formal power series in $n$ variables over $R$. The object of this paper, on one hand, is to relate the prime ideal structure of $R\left[\left[X_{1}, \ldots, X_{n}\right]\right]$ with that of the rings $R_{P}\left[\left[X_{1}, \ldots, X_{n}\right]\right]$ where the $P$ 's are the prime ideals of $R$ and, on the other hand, to relate the prime ideal structure of $R\left[\left[X_{1}, \ldots, X_{n}\right]\right]$ with that of $R\left[X_{1}, \ldots, X_{n}\right]$.

We shall use the symbol mcpil $r$ to mean maximal chain of prime ideals of length $r$. We shall prove the following result:

Proposition. Let $R$ be a Noetherian domain and $P$ a prime ideal of $R$. Then

(1) There exists an mcpil $r$ in $R_{P}\left[\left[X_{1}, \ldots, X_{n}\right]\right]$ if and only if there exists an mcpil $r$ in $R\left[\left[X_{1}, \ldots, X_{n}\right]\right]_{\left(P, X_{1}, \ldots, X_{n}\right)}$.

(2) There exists an mcpil $r$ in $R\left[\left[X_{1}, \ldots, X_{n}\right]\right]_{\left(P, X_{1}, \ldots, X_{n}\right)}$ if and only if there exists an mcpil $r$ in $R\left[X_{1}, \ldots, X_{n}\right)_{\left(P, X_{1} \ldots \ldots X_{n}\right)}$.

Notice that the hypothesis "Noetherian" in the proposition cannot be dropped, nor even be weakened to "locally Noetherian". Indeed, if $R$ is a one-dimensional, non-Noetherian domain such that $R_{P}$ is a rank-one discrete valuation ring for every prime ideal $P \neq(0)$, then $\mathrm{J}$. Arnold has shown that $R[[X]]$ has infinite dimension whereas $R_{P}[[X]]$ has dimension 2 for every prime $P \neq(0)$ and $R[X]$ also has dimension 2 [1, Example 2, p. 303].

In trying to prove the first part of the proposition, one is tempted to take the naive approach of contracting the chains of prime ideals of $R_{P}\left[\left[X_{1}, \ldots, X_{n}\right]\right]$ to $R\left[\left[X_{1}, \ldots, X_{n}\right]\right]$. This approach works in the case of polynomial rings; indeed, the ring $R_{P}\left[X_{1}, \ldots, X_{n}\right]$ being a ring of quotients of $R\left[X_{1}, \ldots, X_{n}\right]$, namely $R_{P}\left[X_{1}, \ldots, X_{n}\right]=R\left[X_{1}, \ldots, X_{n}\right]_{S}$ with $S=R \backslash P$, the contraction from $R_{P}\left[X_{1}, \ldots, X_{n}\right]$ to $R\left[X_{1}, \ldots, X_{n}\right]$ yields a bijection between the maximal chains of prime ideals of length $r$ of $R_{P}\left[X_{1}, \ldots, X_{n}\right]$ and the saturated chains of prime ideals of length $r$ of $R\left[X_{1}, \ldots, X_{n}\right]$ between ( 0$)$ and $\left(P, X_{1}, \ldots, X_{n}\right)$. However, the situation in

Received by the editors November 29, 1982.

1980 Mathematics Subject Classification. Primary 13C15, 13 F25.

Key words and phrases. Chain of prime ideals, formal power series ring, polynomial ring, localization.

(1)1983 American Mathematical Society $0002-9939 / 83 / 0000-1336 / \$ 02.25$ 
the case of power series rings is quite different. If $R$ is a Noetherian domain, $R \neq R_{P}$ and $n \geqslant 1$, then $R_{P}\left[\left[X_{1}, \ldots, X_{n}\right]\right]$ is not a ring of quotients of $R\left[\left[X_{1}, \ldots, X_{n}\right]\right]$ anymore; as a matter of fact, the quotient, field of $R_{P}\left[\left[X_{1}, \ldots, X_{n}\right]\right]$ even has infinite transcendence degree over the quotient field of $R\left[\left[X_{1}, \ldots, X_{n}\right]\right]$ [3, Theorem 1.10, p. 184] and [7, Theorem 3.8, p. 234]. Even worse, a saturated chain of prime ideals of length $r$ in $R_{P}\left[\left[X_{1}, \ldots, X_{n}\right]\right]$ need not contract to a saturated chain of prime ideals of length $r$ in $R\left[\left[X_{1}, \ldots, X_{n}\right]\right]$. This can be seen by the following example that was given to us by J. Arnold.

ExAmple 1 . Let $n \geqslant 2$. Let $R$ be a Noetherian domain and $P$ a prime ideal of $R$ such that $R \neq R_{P}$. Then $R_{P}$ is not integral over $R$ and, consequently, there exist $f_{2}, \ldots, f_{n} \in R_{P}\left[\left[X_{1}\right]\right]$ such that the $R\left[\left[X_{1}\right]\right]$-homomorphism

$$
\varphi: R\left[\left[X_{1}, X_{2}, \ldots, X_{n}\right]\right] \rightarrow R\left[\left[X_{1}, f_{2}, \ldots, f_{n}\right]\right] \subseteq R_{P}\left[\left[X_{1}\right]\right]
$$

defined by $\varphi\left(X_{i}\right)=f_{i}$ for $i \geqslant 2$, is an isomorphism [2, Theorem 3.9, p. 107]. We can extend $\varphi$ to a homomorphism $\varphi^{\prime}: R_{P}\left[\left[X_{1}, X_{2}, \ldots, X_{n}\right]\right] \rightarrow R_{P}\left[\left[X_{1}\right]\right]$ in the obvious way. It is clear that $\varphi^{\prime}$ is surjective, hence that $Q=\operatorname{ker} \varphi^{\prime}$ is a prime ideal of $R_{P}\left[\left[X_{1}, \ldots, X_{n}\right]\right]$ of height $n-1$; moreover $Q \cap R\left[\left[X_{1}, \ldots, X_{n}\right]\right]=(0)$ since the restriction of $\varphi^{\prime}$ to $R\left[\left[X_{1}, \ldots, X_{n}\right]\right]$ is $\varphi$.

This example shows that the naive approach consisting of contracting the chains of prime ideals will not be of any help to prove the first part of the proposition. It will not be of any help either to prove the second part of the proposition. This can be seen by the following example that was also given to us by J. Arnold. The example shows that, contrary to the polynomial ring case, the prime ideals of a chain of length $\geqslant 3$ in $R[[X]]$ can all contract to (0) in $R$, even if $R$ is Noetherian.

EXAMPLE 2 . Let $n \geqslant 1$. Let $k$ be a field, $Y$ an indeterminate over $k$; let $t_{1}, \ldots, t_{n} \in$ $k[[Y]]$ be algebraically independent over $k$ and let $R=k\left[t_{1}, \ldots, t_{n}\right]$. Let $X$ be another indeterminate and consider the $R$-homomorphism $\varphi: R[[X]] \rightarrow k[[Y]]$ defined by

$$
\varphi\left(\sum_{i} f_{i}\left(t_{1}, \ldots, t_{n}\right) X^{i}\right)=\sum_{i} f_{i}\left(t_{1}, \ldots, t_{n}\right) Y^{i} .
$$

It is clear that $\varphi$ is surjective and consequently, that $Q=\operatorname{ker} \varphi$ is a prime ideal of $R[[X]]$ of height $n$; since $\varphi$ is a $R$-homomorphism, we have $Q \cap R=(0)$.

2. Proof of the results. Whenever we talk of a topology on a local ring $(A, M)$, we shall mean the $M$-adic topology.

Proposition. Let $R$ be a Noetherian domain and $P$ a prime ideal of $R$. Then

(1) There exists an mcpil $r$ in $R_{P}\left[\left[X_{1}, \ldots, X_{n}\right]\right]$ if and only if there exists an mcpil $r$ in $R\left[\left[X_{1}, \ldots, X_{n}\right]\right]_{\left(P, X_{1}, \ldots, X_{n}\right)}$.

(2) There exists an mcpil $r$ in $R\left[\left[X_{1}, \ldots, X_{n}\right]\right]_{\left(P, X_{1}, \ldots, X_{n}\right)}$ if and only if there exists an mcpil $r$ in $R\left[X_{1}, \ldots, X_{n}\right]_{\left(P, X_{1}, \ldots, X_{n}\right)}$.

Proof. Notice that each one of the three rings involved in the proposition is a local ring $B$ such that $R\left[X_{1}, \ldots, X_{n}\right]_{\left(P, X_{1}, \ldots, X_{n}\right)}$ is a dense subspace of $B$. Then, the proposition is a consequence of the following theorem. 
THEOREM. Let $R$ be a Noetherian domain and $P$ a prime ideal of $R$. Let $n \geqslant 1$. Let $B$ be a local ring such that $R\left[X_{1}, \ldots, X_{n}\right]_{\left(P, X_{1} \ldots, X_{n}\right)}$ is a dense subspace of $B$. Then, there exists an mcpil $r$ in $R\left[X_{1}, \ldots, X_{n}\right]_{\left(P_{0} X_{1}, \ldots, X_{n}\right)}$ if and only if there exists an mcpil $r$ in $B$.

We first prove that the hypothesis $n \geqslant 1$ is superfluous for one of the implications.

Lemma. Let $A \subseteq B$ be local rings such that $A$ is a dense subspace of $B$. If $A$ has an mcpil $r$, then so does $B$.

Proof. We do an induction on $r$. Let $M$ be the maximal ideal of $A$ and $N$ the maximal ideal of $B$. If $r=0$, then $M$ has height zero, hence $N$ also has height zero and we are through. If $r \geqslant 1$, let $P_{0} \subset P_{1} \subset \cdots \subset P_{r-1} \subset M$ be an mcpil $r$ in $A$. Notice that $A / P_{0}$ is a local domain that has an mcpil $r$ and that it is a dense subspace of $B / P_{0} B$; notice also that if we can show that $B / P_{0} B$ has an mcpil $r$, then $B$ will also have an mcpil $r$ since the height of $P_{0} B$ is equal to the height of $P_{0}$ which is zero [5, Theorem (22.9), p. 75]. This implies that we can suppose that $P_{0}=(0)$ and, consequently, that the height of $P_{1}$ is one. Now, we have that $A / P_{1}$ is a dense subspace of $B / P_{1} B$ and that $A / P_{1}$ has an mcpil $(r-1)$; then, by the hypothesis of induction, $B / P_{1} B$ has an mcpil $(r-1)$, and there exists a saturated chain of prime ideals in $B: Q_{1} \subset Q_{2} \subset \cdots \subset Q_{n-1} \subset N$ where $Q_{1}$ is a minimal prime ideal of $P_{1} B$. Since $P_{1}$ has height 1 , so does $Q_{1}$ and $B$ has an mcpil $r$.

REMARK 1. The converse of the lemma is false in general. Indeed, there exists a two-dimensional local domain $A$ whose completion has an mcpil 1 [5, Example 2, p. 203].

Proof of Theorem. One of the directions is given by the lemma. Now, for the other direction, suppose that $B$ has an mcpil $r$. The completion of $B$, which is the same as the completion of $R\left[X_{1}, \ldots, X_{n}\right]_{\left(P, X_{1}, \ldots, X_{n}\right)}$, is $\hat{R}_{P}\left[\left[X_{1}, \ldots, X_{n}\right]\right]$ where $\hat{R}_{P}$ denotes the completion of $R_{P}$. By the lemma, $\hat{R}_{P}\left[\left[X_{1}, \ldots, X_{n}\right]\right]$ has an mcpil $r$. Since a complete ring is necessarily catenary [5, Theorem (31.1), p. 106], we obtain that $\hat{R}_{P}\left[\left[X_{1}, \ldots, X_{n}\right]\right]$ has a minimal prime ideal of coheight $r$. Then, $R\left[X_{1}, \ldots, X_{n}\right]_{\left(P, X_{1}, \ldots, X_{n}\right)}$ which is equal to $R_{P}\left[X_{1}, \ldots, X_{n}\right]_{\left(P, X_{1}, \ldots, X_{n}\right)}$ has an mcpil $r$ [6, Theorem (2.14), p. 111].

Corollary [4, Theorem 3.1, P. 116]. Let $R$ be a Noetherian domain. Then, the following conditions are equivalent:

(i) $R\left[X_{1}, \ldots, X_{n}\right]$ is catenary.

(ii) $R\left[\left[X_{1}, \ldots, X_{n}\right]\right]$ is catenary.

(iii) $R_{P}\left[\left[X_{1}, \ldots, X_{n}\right]\right]$ is catenary for every maximal ideal $P$ of $R$.

REMARK 2. The result of the lemma is valid more generally if $A \subseteq B$ are local rings for which the theorem of transition holds; the proof goes along the same lines.

\section{REFERENCES}

1. J. T. Arnold, Krull dimension in power series rings, Trans. Amer. Math. Soc. 177 (1973), 299-304.

2. Algebraic extensions of power series rings, Trans. Amer. Math. Soc. 267 (1981), 95-110.

3. J. T. Arnold and D. W. Boyd, Transcendence degree in power series rings, J. Algebra 57 (1979), $180-195$.

4. Y. Lequain, Catenarian property in a domain of formal power series, J. Algebra 65 (1980), 110-117. 
5. M. Nagata, Local rings, Interscience, New York, 1962.

6. L. Ratliff and S. McAdam, Maximal chains of prime ideals in integral extension domains. I, Trans. Amer. Math. Soc. 224 (1976), 103-116.

7. P. B. Sheldon, How changing $D[[X]]$ changes its quotient field, Trans. Amer. Math. Soc. 159 (1971), 223-244.

Universidade Federal do Rio Grande do Sul, Instituto de Matemática, Rua Sarmento Leite 425, 90.000 Porto Alegre, Brazil

Instituto de Matemática Pura e Aplicada, Estrada Dona Castorina 110, 22.460 Rio de JANEIRO, BRAZIL

Current address of both authors: IMPA, Estrada Dona Castorina 110, 22.460 Rio de Janeiro, Brazil 